Forms and Functions of Affective Synchrony

Adrienne Wood ${ }^{1}$, Jennie Lipson $^{1}$, Olivia $_{Z^{2}}{ }^{2}, \&$ Paula Niedenthal $^{2}$

To appear in (Eds.) L. Thomas \& M. Robinson, Embodied Psychology: Thinking, Feeling, and Acting.

1. Department of Psychology, University of Virginia

2. Department of Psychology, University of Wisconsin 


\begin{abstract}
The reproduction of another individual's emotions in the self - the embodiment of perceived emotions - has been demonstrated to constitute one mechanism for emotional information processing. That is, seeing someone's emotion expressions and using one's own face to make the same expression helps the perceiver represent the emotion of the other. When members of a dyad mimic each other's emotion expressions and by consequence converge in their underlying physiology over time we say that the dyad has reached a state of affective synchrony. The present chapter brings together recent theorizing and research on physiological and expressive affective synchrony. We propose affective synchrony serves three interrelated functions: it enables efficient information exchange, allows for interpersonal emotion regulation, and builds social bonds. We review evidence for the contexts in which affective synchrony arises, propose and evaluate the benefits and costs of achieving these states, and end by suggesting paths for future research in this area.
\end{abstract}


Most of social life boils down to moments of connection. People are usually in the business of getting together, staying together, and pursuing behavioral goals together. They flirt, dance, build, converse, play, and even paddle in double kayaks and ride tandem bicycles. Successful connection is not simple, however. At a basic level, acts of connection involve precise temporal and spatial coordination. Furthermore, people are not social agents that can be programmed to coordinate. During interaction real people have emotional responses that sometimes motivate them (e.g., telling them that they do or do not want to continue with an action) and serve to regulate their specific behaviors (e.g., telling them whether to approach or avoid something or someone). Thus, in moments of social connection, two or more people monitor, predict and regulate each other's emotions (e.g., Michael, 2011). How do people accomplish this? How do shared emotions serve to help people get together, stay together and pursue common goals?

As is the case for the emergence of coordinated motor behavior-joint action (Knoblich et al., 2011)—one mechanism for coordinated emotions is an embodied one. As people monitor each other's emotions they tend to produce elements of the perceived emotion in themselves. For example, people make the same dynamic facial expressions as those they observe. The act of mimicry in turn contributes to a cascading activation of the neural states involved in experiencing other components of the emotion (Wood et al., 2016). When two people's emotions align in form as well as temporal dynamics, we can say that a dyad has come into affective synchrony.

The present chapter is about how and why the components of individuals' emotions become synchronous. We will focus our discussion on the synchrony that can emerge within a dyad, although groups of any size can synchronize, and we occasionally point to relevant 
findings about group synchrony. We define synchrony as the dynamic and reciprocal adaptation of the timing of corresponding emotional components of members of interacting dyads. The synchronous state may conform to the mathematical description of an oscillator, with a period, repeating rhythm, or it may take a different shape. In the present chapter we thus call the state of two people's emotions coming into embodied temporal and spatial alignment affective synchrony. Behavioral affective synchrony as we define is somewhat distinct from behavioral mimicry (Lakin \& Chartrand, 2003), which tends to refer to alignment of emergent categories of behavior (e.g., smiling and head scratching) rather than continuous measures. Further, we do not say that people "mimic" each other's physiological states, so we use the term "synchrony" throughout this chapter to unite the discussion of behavioral and physiological variables.

We first review evidence for physiological affective synchrony with a focus on three types of dyads: caretaker-child, romantic partners, and client-therapists. We then make several novel theoretical contributions to the affective synchrony literature. We point out that affective states are not directly transmitted from one person to another-like all forms of information, they require a communication medium. That medium is observable behavior, such as facial expressions, pupillary dilation, and speech. We therefore suggest that observable behavioral cues drive physiological synchrony, particularly when those cues become synchronized across people, which we refer to as expressive affective synchrony. We next integrate evidence in favor of several functions of affective synchrony. These include the proposals that affective synchrony provides 1) a common basis for information processing, 2) an efficient means for finding and meeting challenges and opportunities in the environment, and 3) stronger social connectedness through the generation of feelings of similarity and closeness. We note that affective synchrony sometimes fails to serve these positive functions and can have negative consequences for social 
interactions and relationships depending upon the emotion context, type of social interaction, and individual characteristics. The negative consequences include the maintenance or escalation of both interpersonal conflict and maladaptive affective responding. We end by suggesting where affective synchrony research might most productively go next.

\section{Physiological Synchrony}

Coordinated social interaction involves layers of shared and synchronized physiological processes at multiple timescales (Bietti \& Sutton, 2015; Yun et al., 2012). Conversation partners synchronize their pupillary dilation, suggesting the partners' moment-by-moment changes in attention and arousal are linked (Kang \& Wheatley, 2017). Partners display synchrony in autonomic nervous state measures, such as heart rate (Mitkidis et al., 2015), breathing (Ferrer \& Helm, 2013), skin conductance (Mønster et al., 2016), and cardiac pre-ejection period (West et al., 2017). More global interpersonal neural synchrony occurs during conversation, in terms of both the timing and content of brain activity, reflecting shared understanding (e.g., Stephens et al., 2010). And some social species even synchronize their daily sleep-wake rhythm (Favreau et al., 2009), suggesting physiological synchrony between bonded individuals can extend even to these slower timescales.

Due to its important roles in promoting bonding and emotion regulation (see "Functions of affective synchrony"), many investigations of physiological affective synchrony examine the synchronization of physiological reactivity in mothers and infants. But classic work investigated physiological synchrony in adults, including between clients and their therapists and romantic couples, as well. We focus in this section on research that specifically examines synchronization of autonomic nervous system (ANS) processes such as heart rate, electrodermal activity, respiration and heart rate variability (HRV; for a review of physiological synchrony, see 
Palumbo et al., 2017). These autonomic signals react to alterations in emotional states and covary in different ways, including conforming to oscillating patterns.

\section{Caretaker-Child Synchrony}

Research on mother-infant synchrony was itself inspired by demonstrations that mammalian mothers, through the mechanism of social contact, align their physiological systems with those of their young in order to positively affect the infant's growth (Schanberg et al., 2003) and modulate brain structures implicated in the regulation of stress (Champagne, 2008) and cardiovascular rhythms (Hofer, 1971).

Similarly, human caretakers and infants have been shown to synchronize their cardiac rhythms through the mechanism of touch (Waters et al., 2017) but also through visual and auditory cues (Feldman, 2007). In a study by Feldman and colleagues (Feldman, Magori-Cohen, et al., 2011), cardiac measures of mothers and their 3-month old infants were taken while they engaged in face-to-face interactions, and mothers and infants were also videotaped. Time-series analysis applied to the cardiac output revealed that that mother and infant heart rhythms, but not those of pseudo-dyads, became synchronized with time lags of less than 1 second. Experimenters also coded gaze, expressed affect, and vocal behavior for evidence of synchrony. Analyses relating physiology to these interpersonal behaviors showed further that sychrony in maternal and infant heart rhythms increased during periods of affective and vocal covariation, suggesting the emergence of a broader biobehavioral alignment (Atzil et al., 2012).

Water et al. (2014) observed synchronous heart rate in mothers and infants after mothers had experienced a laboratory stressor. The researchers brought mother and infant pairs into the laboratory and temporarily separated the pairs. During the separation, mothers were randomly assigned to a modified Trier Social Stress test in which they received positive evaluation or 
negative evaluation during the delivery of a speech and a question and answer period. Mothers in a control condition performed the speech task while alone and received no evaluation.

Physiology of mothers was recorded during the task and physiology was recorded in both mother and infant during a reunion period. Results showed that infants' physiological reactivity covaried with mothers' physiological reactivity. Furthermore, the negative-evaluation condition generated higher synchrony in mothers and infants, and this increased over time. Some evidence of synchrony in facilitating social learning was indicated by the finding that the infants of mothers who had been assigned to the two social evaluation conditions later showed more avoidance of strangers in the laboratory (that is, people who might have stressed the mothers) than infants whose mothers were in the control condition.

Caretaker-child physiological synchrony is observed beyond infancy (Main et al., 2016). Woltering et al. (2015) studied the relationship between physiological synchrony and dyadic attunement (or interpersonal responsiveness) in mother-child interactions. Mothers and their 7- to 12-year-old children were invited into the lab to have discussions of both positive and more conflictual topics while being videotaped. Dyadic attunement was manually coded as a sum of signs of engagement, joint attention, and reciprocity. Heart rate of both members of the dyad was measured and physiological synchrony was estimated using a Structural Heteroscedastic Measurement-Error model, which detects linear relationships between two discrete time series. Higher dyadic attunement was observed in positive discussions (i.e., what would you do if you won the lottery) than in a conflictual discussion (i.e., topics of persisting disagreement). Dyadic attunement, at least in smoothly functioning dyads, thus seems to arise more in interactions characterized by positive emotions. Moreover, dyadic attunement predicted higher physiological 
synchrony. This was particularly true during a positive interaction that followed the conflictual one, which suggested to the authors that synchrony positively predicts relationship repair.

\section{Romantic Partner Synchrony}

Physiological synchrony continues over development and has long been documented in romantic couples. In a series of classic studies, for example, Levenson and Gottman (1983) examined sympathetic nervous system (SNS) synchrony between married couples during social interaction tasks. In one, involving a conversation about a negative topic, couples who had identified themselves as high in marital distress showed higher synchrony in heart rate, skin conductance, pulse transmission time and somatic movement than did non-distressed couples (Levenson \& Gottman, 1983). Neither group showed physiological synchrony during a conversation about events of their day. The authors concluded that couples in distress reciprocate negative affect at the physiological level, whereas non-distressed couples do not. However significantly more research on synchrony during couples' interactions has been conducted since, which taken together suggests a more complex view of the presence and function of physiological synchrony in married and romantic partners as discussed further below (Liu et al., 2013; Palumbo et al., 2017; Papp et al., 2013; Timmons et al., 2016; Saxbe \& Repetti, 2010). For instance, Helm et al. (2012) examined the covariation of respiration and heart rate during tasks in which couples gazed into each other's eyes and in which they attempted to mirror each other's physiology. They modeled the physiological signals using coupled linear oscillator models (e.g., Boker \& Nesselroade, 2002). Couple's respiration was found to synchronize during the gaze task and to some degree the imitation task. For heart rate, the findings showed synchrony between couples across both tasks. Similar levels of synchrony were not observed in the patterns of peudo-dyads that were randomly constructed for comparison suggesting that only 
couple's heart rate and respiration are shared during interactions designed to elicit shared emotional arousal. Rather than relating to entrenched conflict, synchrony in these more neutral and positive interactions seems to be associated, as with infants, with dyadic attunement, that is, with positive connection.

\section{Client-Therapist Synchrony}

A similar set of demonstrations have been reported regarding the physiology of clients and therapists during psychotherapy, which is a context in which empathic responding is particularly important (Karvonen et al., 2016; Pascual-Leone, 2009; Ramseyer \& Tschacher, 2006; Ramseyer \& Tschacher, 2011). For instance, Marci et al. (2007) measured skin conductance in psychotherapy clients and their therapists during live clinical encounters. They found that skin conductance alignment during psychotherapy sessions predicted clients' ratings of therapist empathy. Additionally, observer ratings of clips from the sessions indicated that clients and therapists displayed more solidarity and positive regard during moments when their skin conductance concordance was higher.

In a more recent study, Tschacher and Meier (2019) observed significant synchrony between clients and therapists, compared to pseudo-dyads, on multiple physiological indicators including respiration, heart rate and heart rate variability. Synchrony was assessed by both crosscorrelation and concordance (correlation of local slopes) methods, and level of synchrony positively predicted client reports of therapeutic alliance.

\section{Expressive Affective Synchrony}

How does a dyad achieve the embodied state of physiological affective synchrony, when much of the body's physiological state is not outwardly observable? Some instances of physiological synchrony occur because two members of a dyad are attending to the same 
external event: two friends watching a scary movie together will have highly synchronous SNS activity simply because they are responding to the same stimulus. Such parallel affective experiences can still increase feelings of social closeness and liking (Cheong et al., under review). However, this form of synchrony does not even require that partners be able to see, feel, or hear each other, and does not involve mutual causal influence between partners' affective states.

In instances where partners are influenced by each other's physiological states — and not a third variable in the environment - the partners must somehow transmit information about their physiological oscillations (Repp \& Su, 2013). In other words, they must produce observable cues that their partners embody, and such behavioral synchrony then facilitates physiological synchrony (e.g., Oullier et al., 2008). The observable cues may be tactile (Waters et al., 2017; Chatel-Goldman et al., 2014), visual (Schmidt et al., 1990; Schmidt \& O’Brien, 1997), or auditory (Eckland et al., 2019), the latter being particularly powerful synchrony attractors (Repp \& Penel, 2004). The more partners attend to each other's expressive cues, the more likely they are to synchronize with them (Richardson et al., 2007), highlighting the mediating role of communication. Partners may even achieve mutual eye contact in order to increase their partner's attention to their communicative signals, and thereby increase interpersonal synchrony (Leong et al., 2017).

Communication takes two forms: signals and signs (Bradbury \& Vehrencamp, 2011). Signals are communicative behaviors whose primary function is to transmit information between individuals. A soothing spoken utterance intended to calm a restless baby and a scream that alerts group members about a danger are two examples of communicative signals. Communicative signals have predictable effects on a perceiver's physiology, as demonstrated by Martin et al. 
(2018) in a study of the effects of different types of smiles on heart rate and cortisol production. People can also influence the physiology of their interaction partners by producing affectmodulating signs - behaviors that incidentally convey information about the producer's affective state. For instance, shaky hands might be a sign (rather than a signal) of anxiety that can mediate the transfer of arousal from one person to another. Some behavioral cues do not fit neatly on one side of the sign-signal dichotomy: is tearful crying a communicative signal or a sign-like byproduct of physiological dysregulation? But for our purposes, the relevant point is that physiological affective synchrony in a dyad is mediated by the continuous exchange of perceptible signs and/or signals about the partners' internal states.

How much communication is required to maintain affective synchrony in a dyad? Dyads can be more or less similar in their affective and physiological responses to events, perhaps due to personality differences. Vallacher et al. (2005) argue that such similarity will determine how much communication and interpersonal influence the dyad needs to maintain physiological synchrony. Dissimilar dyads need consistent behavioral cues to maintain synchrony, and when communication channels are removed, they will quickly become decoupled. Highly similar dyads, on the other hand, can maintain stable synchrony for more time in the absence of communication.

\section{Demonstrations of Expressive Synchrony}

Behavioral components of emotion, described above, can trigger affective synchrony. For instance, a baby's cry of distress might cause the caregiver to match the distress in some aspects of time and space, even if the caregiver does not (usually) show behavioral synchrony in the sense of bursting into tears. But increasing evidence suggests that dyads do frequently align their bodily expressions of affect, suggesting a system of expressive and physiological synchrony. 
Comprehensive reviews of behavioral synchrony and the closely related phenomenon of nonverbal mimicry (i.e., similarity in form but not timing of expressive behavior) exist elsewhere (e.g., Lakin, 2013; Repp \& Su, 2013; Wheatley et al., 2012). So, we focus here on the conditions under which expressive synchrony is most likely to emerge.

Expressive synchrony is most frequently observed during positive affective interactions. For instance, Riehle et al. (2017) examined the synchrony of activation of smiling and frowning muscles with methods of electromyography (EMG) while participants discussed positive and negative life events. Compared to pseudo-dyads, true dyads synchronized their smiles, as estimated using windowed cross-lagged correlation analysis. Synchronization of smiles was rapid: after one interactant smiled, their partner smiled within 200 to $1000 \mathrm{~ms}$. Interactants' frowns did not appear to synchronize, suggesting that (previously unacquainted and gendermatched) interaction partners are more likely to synchronize positive compared to negative expressions.

Other evidence supports the notion that synchrony tends to accompany positive affect. Likowski et al. (2011) induced happiness or sadness in participants and then exposed them to images of facial expressions. Using EMG to measure participants' facial muscle activity, they found that happy participants were more likely than sad participants to align their faces to match both the positive and negative facial expressions. Mønster et al. (2016) found that smile synchrony within newly formed cooperative teams was correlated with positive team outcomes, while sympathetic nervous system synchrony (measured via skin conductance) was correlated with negative team outcomes. Such findings highlight that not all synchrony is desirable, an idea we return to later. 
Dyads working towards a shared goal that requires coordination are also more likely to align their expressions of emotion. Louwerse et al. (2012) had participants perform a map task (Anderson et al., 1991) while they were videotaped. The task involved unscripted communication about particular routes. Knowledge of the route was distributed between the Instruction Giver, whose maps showed the route, and the Instruction Follower, who had to reproduce the route on a similar map. Maps showed, to different degrees, common landmarks, but some landmarks on the Followers' maps were obscured by grey "inkblots." Coders trained in the Facial Action Coding System (FACS; Ekman et al., 2002) classified participants' facial movements including, of interest here, signs of laughter and smiling, but also manual gestures and features of language. Findings revealed significant synchrony across modalities. In addition, some modalities, including laughter and smiling, showed higher synchrony when landmarks were more extensively obscured by the inkblots, making communication more difficult. The peak latency between one partner producing the behavior and the other partner matching them depended on the behavior, although most latencies were within a few seconds. This suggests that when communicative channels sync in cooperative interactions it provides an embodied grounding for understanding (see also Golland et al., 2019; Chartrand \& Bargh, 1999).

A role for expressive synchrony in communication was demonstrated recently in our laboratory (Zhao et al., under review). Specifically, we tested the prediction that when people interact without the use of spoken language, they become more facially expressive and these expressions become more synchronized in order to compensate for the loss of communication through the verbal channel. Working in pairs, participants took turns completing trials of four different tasks in which they could earn points, awarded to the dyad as a team. Two of the tasks, a risk-taking task and a Jenga tower-building task, were designed to elicit emotion during joint 
action. Importantly, pairs were assigned to either a spoken language permitted or spoken language not permitted condition. As expected, in the latter condition, in which pairs could not use spoken language, both facial expressiveness and also facial expressive synchrony were higher than in the spoken language permitted condition.

In instances of expressive synchrony where there is a clearly-identifiable "leader"-for instance, during unidirectional communication—one might assume that the "synchronizer" is always temporally lagging behind the "leader." However, the synchronizer's expression can predict and precede the onset of the leader's expression. In a documentation of predictive smiling, Heerey and Crossley (2013, Study 2) estimated smile onset asynchronies, or the time lag before an individual returned genuine and polite smiles. Past research has demonstrated that people sometimes return smiles reactively with time lags longer than $200 \mathrm{~ms}$, which is usually conceptualized as facial communicative mimicry (e.g., Wood et al., 2016). The researchers reasoned that synchronization within $200 \mathrm{~ms}$ after smile onset reflects prediction of impending facial expressions in the partner because perceptual processing and subsequent motor output takes longer than $200 \mathrm{~ms}$ (Sanders, 1998). Participants in the study learned to associate neutral faces with key presses, and correct responses were either rewarded with genuine or polite smiles. Participants learned stimulus-response mappings more quickly when the mappings were reinforced with genuine, compared with polite, smiles. In addition, the genuine smiles were returned predictively to a higher extent, suggesting the anticipation of social reward (e.g., a genuine smile), which has been observed in other social learning paradigms (Schultz, 2007). These findings along with those of Riehle et al. (2017), mentioned previously, provide quantitative evidence of predictive facial synchrony. They also support the earlier claim that 
synchrony is more common for positive signals, here manifested as genuine (as opposed to polite) smiles.

What is the relationship between expressive and physiological synchrony? We argued earlier that behavioral cues are the communicative medium through which physiological synchrony is achieved. Expressive synchrony can trigger synchrony of internal states (e.g., physiological synchrony) by continually linking partners' behavior (Feldman, Magori-Cohen, et al., 2011). Expressions are often an embodied manifestation of an internal physiological state and can therefore be an observable and transferrable mediator of physiological synchrony. In a study in which strangers watched videos together, moments of synchronous smiling (measured with facial electromyography) correlated with moments of cardiovascular synchrony (Golland et al., 2019). Expressive synchrony establishes moments of intensive interpersonal coupling and connection. Expressive synchrony, like affective synchrony more broadly, thereby enables information exchange, co-regulation, and social bonding within the dyad. We explore these adaptive consequences next.

\section{Functions of Affective Synchrony}

What is the adaptive function of synchrony? Maybe synchrony has no function and is instead always a by-product of a simpler physical process. For instance, ticking metronomes will fall into sync if they are physically coupled via a shared a surface. But we would not say that the metronomes being in sync has a function. Thankfully, people are more complex than metronomes. Evidence suggests that we reap a number of benefits by connecting with social partners through affective synchrony.

We propose three overlapping but specific adaptive functions of affective synchrony (see Figure 1). First, affective synchrony, like other forms of interpersonal synchrony, reduces the 
number of uncorrelated variables (i.e., complexity) of a dyad, making information processing easier for both partners. In other words, shared affective responses reduce the number of timevarying affective response variables within the dyad from two to one. Second, by sharing their emotional states with others, people are able to detect and respond to challenges and

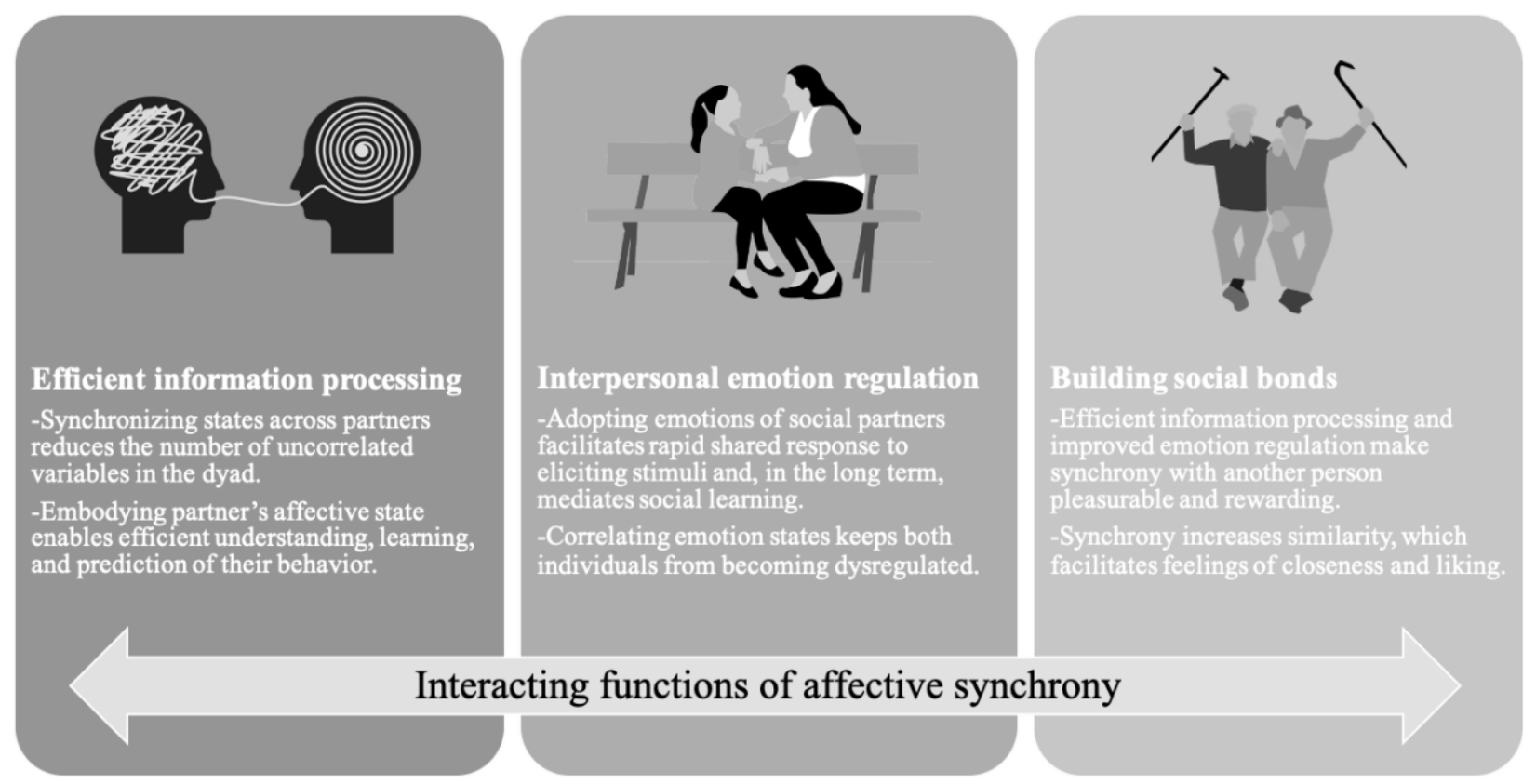

Figure 1. We propose three interconnected levels at which affective synchrony is adaptive.

opportunities in the environment more efficiently. The benefits of this ability can be measured on multiple timescales: in the moment, spouses can pool their resources to co-regulate each other's distress. Caregivers can instill immediate fear and freezing in a baby about to walk off a step by vocalizing their alarm. Over the long-term, affective synchrony enables social learning, as partners align with each other's responses to classes of stimuli that are encountered on repeated occasions. And finally, extensive evidence suggests affective synchrony enhances feelings of similarity and closeness and strengthens social bonds. We explore these three functions next. 


\section{Efficient Information Processing}

We theorize that affective synchrony enables more efficient information processing. Koban et al. (2019) posit that, as a general rule, interpersonal motor synchronization arises as a result of the tendencies of the brain to conserve resources and optimize its representation of the environment. When interacting with a partner, people must represent both their own behavior and internal state and their partner's behavior and internal state. From the outset, the behaviors and internal states of social partners are likely to be similar: People usually choose to affiliate with similar others (e.g., Bahns et al., 2017), and close relational partners tend to share goals, often working together on tasks that require coordination. And if members of an interacting dyad increasingly embody each other's emotional states and behaviors over time, they can jointly represent their own state and actions with the state and actions of their partner. Coupling these self and other representations gives each partner one less thing to keep track of, so interacting synchronously reduces cognitive effort.

Though Koban et al. (2019) focus on spontaneous motor synchronization, the same principles apply to affective synchrony. People use embodied sensorimotor simulation to understand other people's internal states and motivations in addition to their motor actions (Gallese \& Goldman, 1998; Wood et al., 2016). Accordingly, coupling one's own emotion state with the representation of a partner's emotion state is an efficient way to understand the partner's emotions, just as motor synchronization is an efficient way to understand the partner's actions and intentions. Because the partners' emotions are aligned, an individual's own emotional state can be used to predict the future behavior of their partner (Heerey \& Crossley, 2013; Friston \& Frith, 2015; Seth \& Critchley, 2013). For instance, afferent feedback to one's brain from one's smile represents a prediction about a partner's next facial expression and likely social intentions. 
Affective synchrony, in this case, supports interpersonal coordination. By increasing the ease and efficiency of social interactions, synchronization facilitates information processing and enhances communication between interacting partners.

\section{Interpersonal emotion regulation}

As humans, we share almost everything. We share living spaces and communities with people who often share our goals. We feed each other and regulate our temperatures by being close to each other. We share our microbiomes, circadian rhythms, beds, ideas, language, recipes, laundry, beliefs about cosmology, political preferences, and diseases. And according to Social Baseline Theory, the human brain assumes that people have access to social relationships with interdependent others who share our goals and resources (Coan \& Sbarra, 2015). To survive as a species, we have always needed to live and share internal states, possessions, environments, and outcomes with other humans.

Sharing begins in an extreme form: when we are infants, we are helpless and entirely reliant on our caregivers. Human infants cannot regulate their physiology on their own. They depend on caregivers to regulate their diet, temperature, and other aspects of their autonomic function. The infant first experiences physiological and behavioral codependence — and synchrony — with another human while still inside the womb. Physiological and behavioral synchrony between the infant and caregiver continues after birth, as the caregiver is closely attuned to the rhythms and needs of the infant. For instance, parents time their active caregiving to coincide with newborn's sporadic periods of alertness (Feldman, 2012).

Over the course of development, the growing infant learns to associate their behavioral and physiological states with the corresponding states in their caretaker. The infant then learns to reverse the causal relationship in order to synchronize with the caregiver to regulate itself and 
influence the caregiver (Atzil et al., 2019). The tight coupling of physiological and behavioral processes present in an adaptive infant-caregiver relationship (Feldman, Gordon, \& ZagoorySharon, 2011) sets the stage for a lifetime of interpersonal synchrony and co-regulation (this developmental trajectory for synchrony has also been observed in dolphins; Fellner, Bauer, \& Harley, 2006).

Affective synchrony allows us to regulate the behavior of others, particularly when they are unable to regulate themselves (Saxbe et al., 2019). Affective synchrony allows partners to foster context-appropriate emotional states, such as achieving a state of calm prior to falling asleep. According to Social Baseline Theory, a function of the co-regulation of emotion is to render more metabolically efficient the cost of coping with the opportunities and challenges of social life (Coan, 2008; Beckes \& Coan, 2011; Coan, Schaefer, \& Davidson, 2006). Adaptive coregulation does not always mean up-regulating positive states or down-regulating negative states. Sharing negative affect is adaptive in certain contexts; as we have seen, the sharing of stress from the mother transmits an understanding of the presence of threat in the environment to their infant (Waters et al., 2014).

Correlational evidence indirectly supports the notion that affective synchrony enables coregulation of emotion. The degree to which mother-infant dyads achieve behavioral synchrony is positively correlated with the infant's cardiac vagal tone (Porter, 2003). It may not always be the case that the person being regulated is the follower. The regulating person, such as a caregiver, may first match their partner's distress, for instance becoming stressed upon hearing the infant's cry, and then bring the dyad as a single unit back to a low arousal set point.

The concept of co-regulation of affect is central to Butler's (2011) Temporal Interpersonal Emotion System (TIES) model, which proposes that in the context of social 
interactions emotions can be thought of as self-organizing dynamical systems in which the components of emotions emerge and interact across interactants. In the model, co-regulation is operationalized as the bidirectional linkage of oscillating emotional channels between partners, which contributes to emotional stability for both partners (Butler \& Randall, 2013). In a healthy close dyad, the oscillating activity levels in each person's physiological systems are tied to their partner's. If one person's physiological rhythm becomes dysregulated, for instance decoupled from relevant external cues (as in the case of chronic anxiety), their partner's well-calibrated physiological state can regulate them and bring them back to a more adaptive physiological state. For the examples of adult dyads described earlier, including romantic couples and therapeutic alliances, physiological synchrony plays a developmentally appropriate function of contributing to the co-regulation of emotional responses (Liu et al., 2013; Papp et al., 2013; Saxbe \& Repetti, 2010).

In a functioning dyad, partners adjust synchrony according to their own needs and resource availability. The amount of affective synchrony a caregiver-infant dyad achieves is a function of how much co-regulatory support the infant needs and how much co-regulatory support the caregiver is able to provide. A recent study examined the relationship between infant-mother behavioral synchrony and parasympathetic nervous system (PNS) and SNS activity during the mildly stressful still face paradigm (Busuito et al., 2019). Babies with lower PNS activity—infants who might need greater co-regulatory support—elicited more synchronous behavior from their mothers. Mothers who engaged in more synchrony, on the other hand, had higher PNS activity. The authors concluded that these less-aroused mothers were more able to attune to and regulate their babies' distress, perhaps because they had greater emotional resources available to them. 
Affective synchrony does more than allow partners to influence each other's in-themoment affective states. It also helps them teach each other adaptive patterns of emotional responding, which we might consider a long-term manifestation of co-regulation. Social animals use the experiences of others as a source of information about the local environment, for example which foods are safe to eat and which animals are dangerous (Barrett \& Broesch, 2012). If a group of people are in the same environment and have similar goals, it would be inefficient for each one to appraise the environment and its threats and opportunities separately. By yoking their expressions and physiology, they share emotions, which guide and motivate coordinated responses to threats and opportunities. The capacity to synchronize affective responses with others promotes efficient and adaptive responding to stimuli, perhaps before one has even had a chance to appraise them, which is one of the metabolic hallmarks of social learning (Gilbert et al., 2009).

A number of non-human animal groups exhibit the beneficial effects of sharing behavioral responses conferred by embodying each other's expressions and experiences of emotion in the form of affective synchrony. Animals living in groups across phylogeny produce alarm calls when they detect a threat, efficiently spreading their heightened arousal and vigilance to the rest of the group (Snowdon, 2003). The efficiency of this strategy depends on the rest of the group immediately embodying the affective state of the alarm caller - if all group members had to independently detect the threat before acting, it might be too late. Affective states are indeed contagious across animals regardless of whether all group members actually detected the relevant stimulus. For instance, when a stressed zebra fish is placed in a tank with a second nonstressed zebra fish, the fish "average out" their affective states and both become moderately 
stressed (Crane et al., 2018). The fish pool information about the environment by synchronizing their affective states through observable cues that may be visual, olfactory, or otherwise.

Humans similarly share their affective states and the accompanying behavioral responses to evocative stimuli. For instance, as described above, Waters et al. (2014) reunited infants with their mothers after the mothers were exposed to a stressful situation. Infants aligned their states to the heightened arousal state of their mothers, even though they were not directly exposed to the stressor. This affective synchrony was achieved largely through touch (Waters et al., 2017). The adaptive benefits of such affective co-regulation are clear. By sharing her affective response with the infant, the mother is guiding the infant's behavior (see also Vaish \& Striano, 2004). Indeed, the researchers found that the babies behaviorally avoided the person who caused the stress in their mother, even though that person had not done anything to threaten them directly (Waters et al., 2014). The child also learns how to respond to similar stimuli in the future (Skinner et al., 2019). As these examples illustrate, affective synchrony is a general mechanism for social learning (Klinnert et al., 1983).

\section{Building Social Bonds}

As we have shown, synchronizing with a partner is efficient. Merging one's representation of one's own state and actions with one's representation of a partner's state and actions reduces cognitive effort, so partners can process the unfolding interaction with greater ease. In general, processing fluency feels good and leads to positive evaluations (Reber et al., 1998). Because synchronous interactions afford savings in effort and energy consumption, they feel easy and rewarding. People feel good when they are "in sync" with an interaction partner.

Perhaps due to the pleasant feelings that accompany being in sync, synchrony is also linked to positive social outcomes. Behavioral and vocal synchrony increase prosocial behavior, 
perceived social bonding, and positive affect (Mogan et al., 2017). Moving synchronously with social partners leads people to perceive themselves as more similar and increases feelings of compassion and willingness to help (Valdesolo \& DeSteno, 2011). Being in sync also induces feelings of closeness and therefore connection between interacting partners (Sharon-David et al., 2018) and even members of larger groups (e.g., Jackson et al., 2018; Launay et al., 2016).

Of course, many empirical demonstrations linking synchrony to prosociality and rapport rely on brief manipulations in which experimental dyads are led to produce synchronous motor behavior such as tapping, clapping or rocking. We expect that aligning the form and timing of emotional expression and physiology with relational partners in the real world feels at least as intimate as tapping a beat in time with a stranger in a lab experiment. Indeed, Páez et al. (2015) found that people who reported having felt in synchrony with others in naturalistic settings, including a joyful folkloric march and a negatively charged political demonstration, felt greater social support and stronger emotional involvement. Such findings suggest that sharing emotions in real time with another person signals that one's interaction partner is similar to us and likely shares our goals; after all, both are appraising (and preparing to act on) the environment in the same way.

Affective synchrony may also be rewarding because influencing each other's emotional states is pleasurable in itself. When a person smiles and they receive a smile in return, they experience emotional self-agency, or the feeling of having caused the partner's emotions. The process establishes a general feeling of a causal connection and reciprocal influence through cycles of nonverbal communication (e.g., Feldman, 2011). Heightened emotional self-agency has important benefits (Feldman et al., 1999). The benefits can be temporary: When individuals experience themselves as the cause of another's joy, they will likely continue to perform similar 
behaviors. The consequences are also long term: individuals with a general sense of emotional self-agency have higher social and emotional outcomes overall.

In sum, behavioral and affective alignment are pleasurable and cause partners to feel close, motivating them to continue to interact over time. The feelings of ease, self-agency, and rapport experienced during synchronous interactions reward the interacting partners, strengthening the bonds between them.

\section{Costs of Affective Synchrony}

Co-regulation, ease of information processing, and building of social bonds are the proposed functions of affective synchrony. But even functional behaviors can be dysfunctional if they are insensitive to context. Constant, perfect synchrony is maladaptive (Mayo \& Gordon, 2020). After all, if partners perfectly matched each other, they would be unable to benefit from having two brains and bodies rather than one. Moments in which synchrony is beneficial are complemented by moments in which independent behavior is beneficial (Koban et al., 2019; Sebanz et al., 2006; Skewes et al., 2015). Indeed, synchrony tends to occur less than half of the time in a functioning dyad (Feniger-Schaal et al., 2016; Noy et al., 2011).

Mayo and Gordon (2020) refer to dyadic synchrony as a "meta-stable" phenomenon, meaning the dyad does not stably maintain a constant synchronous state, but rather the flexible ability to alternate between synchronous and independent behavior. We have already seen evidence that dyads calibrate their affective synchrony to fit the demands of the interaction (Zhao et al., under review). Danyluck and Page-Gould (2019) demonstrated that PNS and SNS synchrony depends on whether dyads are engaged in a cooperative or competitive context, as well as the extent to which they are allowed to interact (see also Paxton \& Dale, 2013; Miles et al., 2010). 
Affective synchrony within a dyad can be maladaptive when the shared state is negative, and the partners amplify and escalate their unpleasant emotion. In dyads that effectively coregulate each other, the negative emotion of one person is counterbalanced by their partner, returning the dyad to their set point (Reed et al., 2015). Parents, for instance, tend to decrease their physiological arousal when the infant's arousal is heightened (Wass et al., 2019). But in dyads that are dysregulated, the negative emotion of one partner is reinforced by the other's, creating a positive feedback loop of heightened distress.

Growing evidence supports the insight that synchrony during negative affective states is often maladaptive (Saxbe et al., 2019). Romantic partners discussing loss and bereavement showed a negative correlation between heart rate synchrony (indicating shared negative arousal) and feelings of compassion (Corner et al., 2018). In other words, being a supportive partner during a distressing conversation entails not synchronizing with the other person's physiology. Vocal pitch synchrony in client-therapist dyads is associated with worse therapeutic relationships and greater client distress (Reich et al., 2014). In the therapeutic relationship, then, synchrony to achieve and convey empathy must be tempered, lest the therapist amplify the client's distress by reflecting it back to them. Anxiety about the ongoing interaction can also be transmitted from one partner to the other: in a cross-race interaction, a White partner's interracial anxiety can make their Black partner more anxious (West et al., 2017).

While the tendency to match the positive emotions of others is associated with well-being and sympathetic caring, the tendency to match the negative affective states of others is associated with emotional distress and personality disorder symptomatology (Murphy et al., 2018). Mothers with depression synchronize more with their babies during negative states than do mothers without depression (Field et al., 1990). Rather than co-regulating the baby and helping it learn 
how to handle its distress, this dysregulated form of synchrony may heighten and reinforce the baby's distress.

Suveg et al., (2016) measured the heart rate synchrony of mothers and their 3-year-old children during an interactive Etch-a-Sketch task and a control period (in which the pair sat quietly). Physiological synchrony was present during the interactive task and such synchrony predicted the child's ability to self-regulate. Of note, this effect was not observed for a subsample of families that were at high risk, suggesting that the achievement of synchrony can be disruptive to other processes if physiological responses to social contexts are inappropriate or dysregulated.

We posit that context-insensitive affective synchrony is further maladaptive because it disrupts individuals' emotional functioning. The more partners' affective states influence each other, the less they will be influenced by factors outside the dyad. The dyad will therefore be necessarily less emotionally responsive to salient events in the environment. Adaptive emotional responding is flexible and tuned to external events (Schuyler et al., 2014). Preliminary evidence suggests interpersonal synchrony may in fact impede self-regulation (Galbusera et al., 2019). Synchrony can also strengthen in-group bonds to the detriment of intergroup relations, providing further evidence that the benefits are context dependent (Tamborini et al., 2018).

Besides falling into emotion dysregulation, a dyad that is inflexibly synchronized may suffer information processing disadvantages (in contrast to the information processing advantages of synchrony discussed in an earlier section). Social groups afford informational advantages to group members, as the members can crowdsource perception, prior knowledge, and decision-making (Bietti \& Sutton, 2015). But part of those advantages come from the information processing diversity of the group (e.g., Derex \& Boyd, 2016), including diversity in 
their emotional responding. If social partners react identically to all events because they are synchronized, they lose some of that advantageous diversity. Behavioral synchrony in a dyadic problem-solving task was negatively associated with overall performance (Abney et al., 2015). We suggest extreme affective synchrony, beyond mere behavioral synchrony, might have similar drawbacks. In line with this reasoning, Mønster et al. (2016) found that physiological synchrony among cooperating team members was positively related to team cohesion but negatively related to a team's likelihood of adopting a new (potentially more optimal) task behavior over multiple sessions. The teams that synchronized excessively, rather than flexibly switching between synchrony and independence, did not benefit from having multiple minds working to solve a problem.

To summarize, affective synchrony is not always a good thing. When dyads synchronize regardless of context, including during negative affective states, they lose some of the coregulatory and information processing benefits of being social.

\section{Paths for Future Research}

In the present chapter, we have integrated a large body of research that explores a particular form of embodying emotions. Rather than momentary embodiments of emotion, such as an act of facial mimicry, the concept of synchronous emotions entails the matching of expressive cues to emotion and the corresponding matching of physiological activity over real time. We have called "synchronous" those conditions in which the matching of components of emotion represent several mathematical functions including those of covariation and correlation as well as more formal oscillation. However, this matching concept has been named by many different terms in the literature including alignment, entrainment, mimicry, mirroring, imitation and, of course, synchrony. 
Future theorizing, modeling and research will need to formalize the different manners of interpersonal matching of emotions over time. There are almost as many ways to quantify synchrony as there are definitions of the term (e.g., Butler, 2011; Moulder et al., 2018; Thorson et al., 2018). Each has its own assumptions and aims to model slightly different things. A systematic set of quantitative recommendations and an account of their uses and meanings is now sorely needed for progress in further understanding the forms and functions of affective synchrony.

A deeper understanding of the costs and benefits of affective synchrony will require an additional type of modeling which is the modeling of synchrony itself over time. For example, while we have noted that some costs of affective synchrony are observed during states of negative emotion, it could be that synchrony is initially beneficial in supporting the understanding of even another person's negative emotions, but that a divergence from synchrony - a decoupling - is then beneficial because it aids in dyadic emotion co-regulation and the use of contextual information. That is, there may be an important difference between initial synchrony and synchrony that is "stuck" in a negative cycle or state of escalation.

Finally, the precise relationships between the synchronization of different components of emotion, including expressive cues, peripheral physiology and neural states awaits further study.

\section{Conclusion}

The forms and functions of interpersonal synchrony are a rapidly expanding topic of psychological research, thanks to ever-improving measurement and analysis methods (Moulder et al., 2018; Zhao et al., under review). Affective synchrony shares many properties attributed to interpersonal synchrony more generally, such as its potential to reduce interpersonal degrees of freedom (Koban et al., 2019). But affective synchrony also exhibits some unique properties 
compared to pure motor synchrony. It allows dyads to co-regulate each other, learn appropriate emotional responses, engage in empathy-related simulation processes, and strengthen their bonds. And as with other forms of interpersonal synchrony, affective synchrony is most adaptive when dyads flexibly synchronize at relevant moments in an interaction (Mayo \& Gordon, 2020). 


\section{References}

Aarts, H., Custers, R., \& Marien, H. (2009). Priming and authorship ascription: When nonconscious goals turn into conscious experiences of self-agency. Journal of Personality and Social Psychology, 96(5), 967-979. https://doi.org/10.1037/a0015000

Abney, D., Paxton, A., Dale, R., \& Kello, C. (2015). Movement dynamics reflect a functional role for weak coupling and role structure in dyadic problem solving. Cognitive Processing, 16(4), 325-332.

Anderson, A. H., Bader, M., Bard, E. G., Boyle, E., Doherty, G. M., Garrod, S., Isard, S. D., Kowtko, J. C., McAllister, J., Miller, J., Sotillo, C. F., Thompson, H.S., Weinart, R. (1991). The HCRC Map Task Corpus. Language and Speech 34(4), 351-366.

Assaneo, M. F., Ripollés, P., Orpella, J., Lin, W. M., de Diego-Balaguer, R., \& Poeppel, D. (2019). Spontaneous synchronization to speech reveals neural mechanisms facilitating language learning. Nature Neuroscience, 22(4), 627-632.

Atzil, S., Gao, W., Fradkin, I., \& Barrett, L. F. (2018). Growing a social brain. Nature Human Behaviour, 2(9), 624-636.

Atzil, S., Hendler, T., Zagoory-Sharon, O., Winetraub, Y., \& Feldman, R. (2012). Synchrony and specificity in the maternal and the paternal brain: relations to oxytocin and vasopressin. Journal of the American Academy of Child \& Adolescent Psychiatry, 51(8), 798-811.

Augello, A., Infantino, I., Maniscalco, U., Pilato, G., \& Vella, F. (2017, April). The effects of soft somatosensory system on the execution of robotic tasks. In 2017 First IEEE International Conference on Robotic Computing (IRC) (pp. 14-21). IEEE.

Bahns, A. J., Crandall, C. S., Gillath, O., Preacher, K. J. (2017). Similarity in relationships as niche construction: Choice, stability, and influence within dyads in a free choice 
environment. Journal of Personality and Social Psychology, 112, 329-355.

doi:10.1037/pspp0000088

Baltrušaitis, T., Mahmoud, M., \& Robinson, P. (2015). Cross-dataset learning and personspecific normalisation for automatic Action Unit detection. 2015 11th IEEE International Conference and Workshops on Automatic Face and Gesture Recognition (FG), 06, 1-6. https://doi.org/10.1109/FG.2015.7284869

Barrett, H. C., \& Broesch, J. (2012). Prepared social learning about dangerous animals in children. Evolution and Human Behavior, 33(5), 499-508.

Beckes, L. \& Coan, J. A. (2011). Social baseline theory: The role of social proximity in emotion and economy of action. Social and Personality Psychology Compass, 5, 976-988.

Berman, J. S., Dale, R., \& Levitt, H. M. (2014). Vocal synchrony in psychotherapy. Journal of Social and Clinical Psychology, 33(5), 481-494. https://doi.org/10.1521/jscp.2014.33.5.481

Bietti, L. M., \& Sutton, J. (2015). Interacting to remember at multiple timescales: Coordination, collaboration, cooperation and culture in joint remembering. Interaction Studies, 16(3), 419-450. doi:10.1075/is.16.3.04bie

Boker, S. M., \& Nesselroade, J. R. (2002). A method for modeling the intrinsic dynamics of intraindividual variability: Recovering the parameters of simulated oscillators in multi-wave panel data. Multivariate Behavioral Research, 37(1), 127-160.

Bradbury, J. W., \& Vehrencamp, S. L. (2011). Principles of animal communication (Second Edition). Oxford: Oxford University Press.

Busuito, A., Quigley, K. M., Moore, G. A., Voegtline, K. M., \& DiPietro, J. A. (2019). In sync: Physiological correlates of behavioral synchrony in infants and mothers. Developmental Psychology, 55(5), 1034-1045. https://doi.org/10.1037/dev0000689 
Butler, E. A. (2011). Temporal interpersonal emotion systems: The "TIES" that form relationships. Personality and Social Psychology Review, 15(4), 367-393.

Butler, E. A. (2017). Emotions are temporal interpersonal systems. Current Opinion in Psychology, 17, 129-134.

Butler, E. A., \& Randall, A. (2013). Emotional coregulation in close relationships. Emotion Review, 5(2), 202-210.

Carr, K. W., White-Schwoch, T., Tierney, A. T., Strait, D. L., \& Kraus, N. (2014). Beat synchronization predicts neural speech encoding and reading readiness in preschoolers. Proceedings of the National Academy of Sciences, 111(40), 14559-14564.

Champagne, F. A. (2008). Epigenetic mechanisms and the transgenerational effects of maternal care. Frontiers in. Neuroendocrinology, 29(3), 386-397.

Chartrand, T. L., \& Bargh, J. A. (1999). The chameleon effect: The perception-behavior link and social interaction. Journal of Personality and Social Psychology, 76, 893-910.

Chatel-Goldman, J., Congedo, M., Jutten, C., \& Schwartz, J. L. (2014). Touch increases autonomic coupling between romantic partners. Frontiers in Behavioral Neuroscience, 8 , 95.

Cheong, J., Molani, Z., Sadhukha, S., \& Chang, L. J. (under review). Synchronized affect in shared experiences strengthens social connection. https://doi.org/10.31234/osf.io/bd9wn

Churamani, N., Kerzel, M., Strahl, E., Barros, P., \& Wermter, S. (2017, May). Teaching emotion expressions to a human companion robot using deep neural architectures. In 2017 International Joint Conference on Neural Networks (IJCNN)(pp. 627-634). IEEE. 
Coan, J. A. (2008). Toward a neuroscience of attachment. In J. Cassidy and P. R. Shaver (Eds.) Handbook of attachment: Theory, research, and clinical applications, 2nd edition (pp. 241265). New York: Guilford Press.

Coan, J. A., \& Sbarra, D. A. (2015). Social baseline theory: The social regulation of risk and effort. Current Opinion in Psychology, 1, 87-91.

Coan, J. A., Schaefer, H. S., \& Davidson, R. J. (2006). Lending a hand social regulation of the neural response to threat. Psychological Science, 17, 1032-1039.

Corner, G. W., Saxbe, D. E., Chaspari, T., Rasmussen, H. F., Perrone, L., Pettit, C., Friendly, M., Timmons, A. C., \& Margolin, G. (2018). Compassion in a heartbeat: Physiology during couples' loss discussions. Journal of Social and Personal Relationships. doi: $10.1177 / 0265407518770267$.

Crane, A. L., Bairos-Novak, K. R., Sacco, L. H., \& Ferrari, M. C. O. (2018). The socially mediated recovery of a fearful fish paired with periodically replaced calm models. Proceedings of the Royal Society B: Biological Sciences, 285(1888), 20180739. doi: $10.1098 / \mathrm{rspb} .2018 .0739$

Dale, R., Fusaroli, R., Duran, N. D., \& Richardson, D. C. (2013). The Self-Organization of Human Interaction. In Psychology of Learning and Motivation (Vol. 59, pp. 43-95). Elsevier. doi: $\underline{10.1016 / \mathrm{B} 978-0-12-407187-2.00002-2}$

Danyluck, C., \& Page-Gould, E. (2019). Social and Physiological Context can Affect the Meaning of Physiological Synchrony. Scientific Reports, 9(1), 8222. doi: 10.1038/s41598$\underline{019-44667-5}$ 
Derex, M., \& Boyd, R. (2016). Partial connectivity increases cultural accumulation within groups. Proceedings of the National Academy of Sciences, 113(11), 2982-2987. doi: $10.1073 /$ pnas. 1518798113

Eckland, N. S., Leyro, T. M., Mendes, W. B., \& Thompson, R. J. (2019). The Role of Physiology and Voice in Emotion Perception During Social Stress. Journal of Nonverbal Behavior, 43(4), 493-511.

Ekman, P., Friesen, W. V., \& Hager, J. C. (2002). Facial Action Coding System. Manual and Investigator's Guide. Salt Lake City, UT Research Nexus.

Favreau, A., Richard-Yris, M. A., Bertin, A., Houdelier, C., \& Lumineau, S. (2009). Social influences on circadian behavioural rhythms in vertebrates. Animal Behaviour, 77(5), 983989.

Feldman, R. (2007). Maternal-infant contact and child development: Insights from the kangaroo intervention. In L. L'Abate (Ed.), Low-cost approaches to promote physical and mental health: Theory, research, and practice (p. 323-351). Springer Science + Business Media. https://doi.org/10.1007/0-387-36899-X_16

Feldman, R. (2011). Maternal touch and the developing infant. In M. J. Hertenstein \& S. J. Weiss (Eds.), The handbook of touch: Neuroscience, behavioral, and health perspectives (p. 373-407). Springer Publishing Co.

Feldman, R. (2012). Bio-behavioral synchrony: A model for integrating biological and microsocial behavioral processes in the study of parenting. Parenting, 12, 154-164.

Feldman, R. (2014). Synchrony and the neurobiological basis of social affiliation. In M. Mikulincer \& P. R. Shaver (Eds.), The Herzliya series on personality and social psychology. Mechanisms of social connection: From brain to group (pp. 145-166). 
Feldman, R. (2017). The neurobiology of human attachments. Trends in Cognitive Sciences, 21(2), 80-99.

Feldman, R., Magori-Cohen, R., Galili, G., Singer, M., \& Louzoun, Y. (2011). Mother and infant coordinate heart rhythms through episodes of interaction synchrony. Infant Behavior and Development, 34(4), 569-577.

Feldman, R., Greenbaum, C. W., \& Yirmiya, N. (1999). Mother-infant affect synchrony as an antecedent of the emergence of self-control. Developmental Psychology, 35(1), 223.

Feldman, R., Gordon, I., \& Zagoory-Sharon, O. (2011). Maternal and paternal plasma, salivary, and urinary oxytocin and parent-infant synchrony: considering stress and affiliation components of human bonding. Developmental Science, 14(4), 752-761.

Fellner, W., Bauer, G. B., \& Harley, H. E. (2006). Cognitive implications of synchrony in dolphins: A review. Aquatic Mammals, 32(4), 511.

Feniger-Schaal, R., Noy, L., Hart, Y., Koren-Karie, N., Mayo, A. E., \& Alon, U. (2016). Would you like to play together? Adults' attachment and the mirror game. Attachment \& Human Development, 18(1), 33-45. https://doi.org/10.1080/14616734.2015.1109677

Ferrer, E., \& Helm, J. L. (2013). Dynamical systems modeling of physiological coregulation in dyadic interactions. International Journal of Psychophysiology, 88(3), 296-308.

Field, T., Healy, B. T., Goldstein, S., \& Guthertz, M. (1990). Behavior-state matching and synchrony in mother-infant interactions of nondepressed versus depressed dyads. Developmental Psychology, 26(1), 7-14. doi:10.1037/0012-1649.26.1.7

Friston, K. J., \& Frith, C. D. (2015). A duet for one. Consciousness and Cognition, 36, 390-405. doi:10.1016/j.concog.2014.12.003 
Galbusera, L., Finn, M. T. M., Tschacher, W., \& Kyselo, M. (2019). Interpersonal synchrony feels good but impedes self-regulation of affect. Scientific Reports, 9(1), 14691. doi:10.1038/s41598-019-50960-0

Gallese, V., \& Goldman, A. (1998). Mirror Neurons and the Simulation Theory of MindReading. Trends in Cognitive Sciences, 2, 493-501.

Gilbert, D. T., Killingsworth, M. A., Eyre, R. N., \& Wilson, T. D. (2009). The Surprising Power of Neighborly Advice. Science, 323(5921), 1617-1619. doi:10.1126/science.1166632

Golland, Y., Mevorach, D., Levit-Binnun, N., 2019. Affiliative zygomatic synchrony in copresent strangers. Scientific Reports, 9(1), 3120.

Heerey, E. A., \& Crossley, H. M. (2013). Predictive and reactive mechanisms in smile reciprocity. Psychological Science, 24(8), 1446-1455.

Helm, J. L., Sbarra, D., \& Ferrer, E. (2012). Assessing cross-partner associations in physiological responses via coupled oscillator models. Emotion, 12(4), 748-762.

Hofer, M. A. (1971). Cardiac rate regulated by nutritional factor in young rats. Science, 172, 1039-1041.

Hoque, M. E., Yeasin, M., \& Louwerse, M. M. (2006, August). Robust recognition of emotion from speech. In International Workshop on Intelligent Virtual Agents (pp. 42-53). Springer, Berlin, Heidelberg.

Jackson, J. C., Jong, J. Bilkey, D., Whitehouse, H., Zollmann, S., McNaughton, C., Halberstadt, J. (2018). Synchrony and physiological arousal increase cohesion and cooperation in large naturalistic groups. Scientific Reports, 8, 127. 
Kang, O., \& Wheatley, T. (2017). Pupil dilation patterns spontaneously synchronize across individuals during shared attention. Journal of Experimental Psychology: General, 146, $569-576$.

Kimura, M., \& Daibo, I. (2006). Interactional Synchrony in Conversations about Emotional Episodes: A Measurement by "the Between-Participants Pseudosynchrony Experimental Paradigm". Journal of Nonverbal Behavior, 30(3), 115-126.

Klinnert, M. D., Campos, J. J., Sorce, J. F., Emde, R. N., \& Svejda, M. (1983). Emotions as behavior regulators: Social referencing in infancy. In Emotions in Early Development (pp. 57-86). Elsevier. doi:10.1016/B978-0-12-558702-0.50009-1

Knoblich, G., Butterfill, S., \& Sebanz, N. (2011). Psychological research on joint action: theory and data. In Psychology of learning and motivation (Vol. 54, pp. 59-101). Academic Press.

Koban, L., \& Pourtois, G. (2014). Brain systems underlying the affective and social monitoring of actions: An integrative review. Neuroscience and Biobehavioral Reviews, 46(1), 71-84.

Koban, L., Ramamoorthy, A., \& Konvalinka, I. (2019). Why do we fall into sync with others? Interpersonal synchronization and the brain's optimization principle. Social Neuroscience, 14(1), 1-9. doi: $10.1080 / 17470919.2017 .1400463$

Koschate, M., Potter, R., Bremner, P., \& Levine, M. (2016, March). Overcoming the uncanny valley: Displays of emotions reduce the uncanniness of humanlike robots. In The Eleventh ACM/IEEE International Conference on Human Robot Interaction (pp. 359-365). IEEE Press.

Karvonen, A., Kykyri, V. L., Kaartinen, J., Penttonen, M., \& Seikkula, J. (2016). Sympathetic nervous system synchrony in couple therapy. Journal of Marital and Family Therapy, 42(3), 383-395. 
Lakin, J. L. (2013). Behavioral mimicry and interpersonal synchrony. In Nonverbal Communication (pp. 539-575). Boston, MA, US: De Gruyter Mouton. doi: $10.1515 / 9783110238150.539$

Lakin, J. L., \& Chartrand, T. L. (2003). Using nonconscious behavioral mimicry to create affiliation and rapport. Psychological Science, 14(4), 334-339. doi:10.1111/14679280.14481.

Launay, J., Tarr, B., \& Dunbar, R. I. M. (2016). Synchrony as an adaptive mechanism for largescale human social bonding. Ethology, 122(10), 779-789. https://doi.org/10.1111/eth.12528

Lee, D. H., \& Anderson, A. K. (2017). Reading what the mind thinks from how the eye sees. Psychological Science, 28(4), 494-503.

Lee, C. C., Black, M., Katsamanis, A., Lammert, A. C., Baucom, B. R., Christensen, A., ... \& Narayanan, S. S. (2010). Quantification of prosodic entrainment in affective spontaneous spoken interactions of married couples. In Eleventh Annual Conference of the International Speech Communication Association.

Lee, D. H., Mirza, R., Flanagan, J. G., \& Anderson, A. K. (2014). Optical origins of opposing facial expression actions. Psychological Science, 25(3), 745-752.

Leong, V., Byrne, E., Clackson, K., Georgieva, S., Lam, S., \& Wass, S. (2017). Speaker gaze increases information coupling between infant and adult brains. Proceedings of the National Academy of Sciences, 201702493. doi:10.1073/pnas.1702493114

Levenson, R. W., \& Gottman, J. M. (1983). Marital interaction: Physiological linkage and affective exchange. Journal of Personality and Social Psychology, 45(3), 587-597. 
Likowski, K. U., Weyers, P., Seibt, B., Stöhr, C., Pauli, P., \& Mühlberger, A. (2011). Sad and Lonely? Sad Mood Suppresses Facial Mimicry. Journal of Nonverbal Behavior, 35(2), 101117. doi: $10.1007 / \mathrm{s} 10919-011-0107-4$

Liu, S., Rovine, M. J., Cousino Klein, L., \& Almeida, D. M. (2013). Synchrony of diurnal cortisol pattern in couples. Journal of Family Psychology, 27(4), 579-588.

Lundqvist, D., Esteves, F., \& Ohman, A. (1999). The face of wrath: Critical features for conveying facial threat. Cognition \& Emotion, 13(6), 691-711.

Lopez-Rincon, A. Emotion Recognition using Facial Expressions in Children using the NAO Robot. In 2019 International Conference on Electronics, Communications and Computers (CONIELECOMP) (pp. 146-153). IEEE.

Louwerse, M. M., Dale, R., Bard, E. G., \& Jeuniaux, P. (2012). Behavior matching in multimodal communication is synchronized. Cognitive Science, 36(8), 1404-1426.

Main, A., Paxton, A., \& Dale, R. (2016). An exploratory analysis of emotion dynamics between mothers and adolescents during conflict discussions. Emotion, 16(6), 913.

Marci, C. D., Ham, J., Moran, E., \& Orr, S. P. (2007). Physiologic correlates of perceived therapist empathy and social-emotional process during psychotherapy. Journal of Nervous and Mental Disease, 195(2), 103-111.

Marcos, S., Gómez-García-Bermejo, J., Zalama, E., \& López, J. (2011, May). Nonverbal communication with a multimodal agent via facial expression recognition. In 2011 IEEE International Conference on Robotics and Automation (pp. 1199-1204). IEEE.

Martin, J. D., Abercrombie, H. C., Gilboa-Schechtman, E., \& Niedenthal, P. M. (2018). Functionally distinct smiles elicit different physiological responses in an evaluative context. Scientific Reports, 8(1), 1-8. 
Mayo, O., \& Gordon, I. (2020). In and out of synchrony-Behavioral and physiological dynamics of dyadic interpersonal coordination. Psychophysiology, e13574. doi:10.1111/psyp.13574

Michael, J. (2011). Shared emotions and joint action. Review of Philosophy and Psychology, 2(2), 355-373.

Miles, L. K., Griffiths, J. L., Richardson, M. J., \& Macrae, C. N. (2010). Too late to coordinate: Contextual influences on behavioral synchrony. European Journal of Social Psychology, 40(1), 52-60. https://doi.org/10.1002/ejsp.721

Mitkidis, P., McGraw, J. J., Roepstorff, A., \& Wallot, S. (2015). Building trust: Heart rate synchrony and arousal during joint action increased by public goods game. Physiology \& Behavior, 149, 101-106.

Mogan, R., Fischer, R., \& Bulbulia, J. A. (2017). To be in synchrony or not? A metaanalysis of synchrony's effects on behavior, perception, cognition and affect. Journal of Experimental Social Psychology, 72, 13-20. http://dx.doi.org/10.1016/j.jesp.2017. 03.009.

Mønster, D., Håkonsson, D. D., Eskildsen, J. K., \& Wallot, S. (2016). Physiological evidence of interpersonal dynamics in a cooperative production task. Physiology \& Behavior, 156, 2434.

Moulder, R. G., Boker, S. M., Ramseyer, F., \& Tschacher, W. (2018). Determining synchrony between behavioral time series: An application of surrogate data generation for establishing falsifiable null-hypotheses. Psychological Methods, 23(4), 757.

Murphy, B. A., Costello, T. H., \& Lilienfeld, S. O. (2018). Is empathic contagion helpful or harmful? Overlooked heterogeneity in the Empathy Index. Psychological Assessment, 30(12), 1703-1708. https://doi.org/10.1037/pas0000641 
Newman, B., \& Newman, P. (2009). Development through life: A psychosocial approach. (10th ed.) Belmont, CA: Wadsworth, Cengage Learning.

Noy, L., Dekel, E., \& Alon, U. (2011). The mirror game as a paradigm for studying the dynamics of two people improvising motion together. Proceedings of the National Academy of Sciences, 108, 20947-20952. doi:10.1073/pnas.1108155108

Oullier, O., de Guzman, G. C., Jantzen, K. J., Lagarde, J., \& Kelso, J. A. S. (2008). Social coordination dynamics: Measuring human bonding. Social Neuroscience, 3(2), 178-192.

Otero, M. C., Wells, J. L., Chen, K. H., Brown, C. L., Connelly, D. E., Levenson, R. W., \& Fredrickson, B. L. (2019). Behavioral indices of positivity resonance associated with longterm marital satisfaction. Emotion.

Páez, D., Rimé, B., Basabe, N., Wlodarczyk, A., \& Zumeta, L. (2015). Psychosocial effects of perceived emotional synchrony in collective gatherings. Journal of Personality and Social Psychology, 108(5), 711.

Palumbo, R. V., Marraccini, M. E., Weyandt, L. L., Wilder-Smith, O., McGee, H. A., Liu, S., \& Goodwin, M. S. (2017). Interpersonal autonomic physiology: A systematic review of the literature. Personality and Social Psychology Review, 21(2), 99-141. https:// doi.org/10.1177/1088868316628405

Papp, L. M., Pendry, P., Simon, C. D., \& Adam, E. K. (2013). Spouses' cortisol associations and moderators: Testing physiological synchrony and connectedness in everyday life. Family Process, 52(2), 284-298.

Pascual-Leone, A. (2009). Dynamic emotional processing in experiential therapy: Two steps forward, one step back. Journal of Consulting and Clinical Psychology, 77, 113-126. 
Paxton, A., \& Dale, R. (2013). Argument disrupts interpersonal synchrony. The Quarterly Journal of Experimental Psychology, 66(11), 2092-2102. https://doi.org/10.1080/17470 218.2013.853089

Porter, C. L. (2003). Coregulation in Mother-Infant Dyads: Links to Infants' Cardiac Vagal Tone. Psychological Reports, 92(1), 307-319. doi:10.2466/pr0.2003.92.1.307

Ramseyer, F., \& Tschacher, W. (2006). Synchrony: A core concept for a constructivist approach to psychotherapy. Constructivism in the Human Sciences, 11(1-2), 150-171.

Ramseyer, F., \& Tschacher, W. (2011). Nonverbal synchrony in psychotherapy: Coordinated body movement reflects relationship quality and outcome. Journal of Consulting and Clinical Psychology, 79(3), 284-295.

Reber, R., Winkielman, P., \& Schwarz, N. (1998). Effects of perceptual fluency on affective judgments. Psychological Science, 9(1), 45-48.

Reed, R. G., Barnard, K., \& Butler, E. A. (2015). Distinguishing emotional co-regulation from co-dysregulation: An investigation of emotional dynamics and body-weight in romantic couples. Emotion, 15, 45-60. doi: http://dx.doi.org/10.1037/a0038561

Repp, B. H., \& Penel, A. (2004). Rhythmic movement is attracted more strongly to auditory than to visual rhythms. Psychological Research, 68, 252-270.

Repp, B. H., \& Su, Y.-H. (2013). Sensorimotor synchronization: A review of recent research (2006-2012). Psychonomic Bulletin \& Review, 20(3), 403-452.

Richardson, M. J., Marsh, K. L., Isenhower, R. W., Goodman, J. R. L., \& Schmidt, R. C. (2007). Rocking together: Dynamics of intentional and unintentional interpersonal coordination. Human Movement Science, 26(6), 867-891. doi:10.1016/j. humov.2007.07.002 
Riehle, M., Kempkensteffen, J., \& Lincoln, T. M. (2017). Quantifying facial expression synchrony in face-to-face dyadic interactions: Temporal dynamics of simultaneously recorded facial EMG signals. Journal of Nonverbal Behavior, 41(2), 85-102.

Reich, C. M., Berman, J. S., Dale, R., \& Levitt, H. M. (2014). Vocal synchrony in psychotherapy. Journal of Social and Clinical Psychology, 33(5), 481-494.

Sanders, A. F. (1998). Elements of human performance: Reaction processes and attention in human skill. Mahwah, NJ: Erlbaum.

Saxbe, D. E., Beckes, L., Stoycos, S. A., \& Coan, J. A. (2019). Social Allostasis and Social Allostatic Load: A New Model for Research in Social Dynamics, Stress, and Health. Perspectives on Psychological Science, 1745691619876528.

Saxbe, D., \& Repetti, R. L. (2010). For better or worse? Coregulation of couples' cortisol levels and mood states. Journal of Personality and Social Psychology, 98(1), 92-103.

Schanberg, S. M., Ingledue, V. F., Lee, J. Y., Hannun, Y. A., \& Bartolome, J. V. (2003). PKC alpha mediates maternal touch regulation of growth-related gene expression in infant rats. Neuropsychopharmacology, 28, 1026-1030.

Schmidt, R. C., Carello, C., \& Turvey, M. T. (1990). Phase transitions and critical fluctuations in the visual coordination of rhythmic movements between people. Journal of Experimental Psychology: Human Perception and Performance, 16, 2.

Schmidt, R. C., \& O'Brien, B. (1997). Evaluating the dynamics of unintended interpersonal coordination. Ecological Psychology, 9(3), 189-206. doi:10.1207/s15326969eco0903_2

Schultz, W. (2007). Multiple dopamine functions at different time courses. Annual Review of Neuroscience, 30, 259-288. doi:10.1146/annurev.neuro.28.061604.135722 
Schuyler, B. S., Kral, T. R. A., Jacquart, J., Burghy, C. A., Weng, H. Y., Perlman, D. M., ... Davidson, R. J. (2014). Temporal dynamics of emotional responding: Amygdala recovery predicts emotional traits. Social Cognitive and Affective Neuroscience, 9(2), 176-181. doi: $\underline{10.1093 / \text { scan/nss131 }}$

Sebanz, N., Bekkering, H., \& Knoblich, G. (2006). Joint action: Bodies and minds moving together. Trends in Cognitive Sciences, 10(2), 70-76. doi:10.1016/j.tics.2005.12.009

Seth, A. K., \& Critchley, H. D. (2013). Extending predictive processing to the body: Emotion as interoceptive inference. Behavioral and Brain Sciences, 36(3), 227-228. http://dx.doi.org/10.1017/S0140525X12002270

Sharon-David, H., Mizrahi, M., Rinott, M., Golland, Y., \& Birnbaum, G. E. (2018). Being on the same wavelength: Behavioral synchrony between partners and its influence on the experience of intimacy. Journal of Social and Personal Relationships, 0265407518809478. doi:10.1177/0265407518809478

Shore, D. M., Heerey, E. A. (2011). The value of genuine and polite smiles. Emotion, 11, 169174. doi:10.1037/a0022601

Skewes, J. C., Skewes, L., Michael, J., \& Konvalinka, I. (2015). Synchronised and complementary coordination mechanisms in an asymmetric joint aiming task. Experimental Brain Research, 233(2), 551-565. doi:10.1007/s00221-014-4135-2

Skinner, A. L., Osnaya, A., Patel, B., \& Perry, S. P. (2019). Mimicking Others’ Nonverbal Signals is Associated with Increased Attitude Contagion. Journal of Nonverbal Behavior. doi:10.1007/s10919-019-00322-1 
Snowdon, C. T. (2003). Expression of emotion in nonhuman animals. In R. J. Davidson, K. R. Scherer, \& H. H. Goldsmith (Eds.), Series in affective science. Handbook of affective sciences (p. 457-480). Oxford University Press.

Sobol-Shikler, T., \& Robinson, P. (2009). Classification of complex information: Inference of co-occurring affective states from their expressions in speech. IEEE Transactions on Pattern Analysis and Machine Intelligence, 32(7), 1284-1297.

Susskind, J. M., \& Anderson, A. K. (2008). Facial expression form and function. Communicative \& Integrative Biology, 1(2), 148-149.

Suveg, C., Shaffer, A., \& Davis, M. (2016). Family stress moderates relations between physiological and behavioral synchrony and child self-regulation in mother-preschooler dyads. Developmental Psychobiology, 58(1), 83-97. https://doi.org/10.1002/dev.21358

Suzuki, N., \& Katagiri, Y. (2007). Prosodic alignment in human-computer interaction. Connection Science, 19(2), 131-141.

Stephens, G. J., Silbert, L. J., \& Hasson, U. (2010). Speaker-listener neural coupling underlies successful communication. Proceedings of the National Academy of Sciences, 107(32), $14425-14430$.

Tamborini, R., Novotny, E., Prabhu, S., Hofer, M., Bente, G., Grall, C., Klebig, B., Hahn, L., Slaker, J., Ratan, R. (2018). The effect of behavioral synchrony with black or white virtual agents on outgroup trust. Computers in Human Behavior, 83, 176-183.

doi:10.1016/j.chb.2018.01.037

Thorson, K. R., West, T. V., \& Mendes, W. B. (2018). Measuring physiological influence in dyads: A guide to designing, implementing, and analyzing dyadic physiological studies. Psychological Methods, 23(4), 595-616. doi:10.1037/met0000166 
Timmons, A. C., Margolin, G., \& Saxbe, D. E. (2015). Physiological linkage in couples and its implications for individual and interpersonal functioning: A literature review. Journal of Family Psychology, 29(5), 720-731.

Tschacher, W., \& Meier, D. (2019). Physiological synchrony in psychotherapy sessions. Psychotherapy Research, 1-16.

Vaish, A., \& Striano, T. (2004). Is visual reference necessary? Contributions of facial versus vocal cues in 12-month-olds' social referencing behavior. Developmental Science, 7(3), 261-269. doi:10.1111/j.1467-7687.2004.00344.x

Valdesolo, P., \& DeSteno, D. (2011). Synchrony and the social tuning of compassion. Emotion, 11(2), 262-266. https://doi.org/10.1037/a0021302

Vallacher, R. R., Nowak, A., \& Zochowski, M. (2005). Dynamics of social coordination: The synchronization of internal states in close relationships. Interaction Studies, 6(1), 35-52. https://doi. org/10.1075/is.6.1.04val

van der Schalk, J., Fischer, A. H., Doosje, B., Wigboldus, D., Hawk, S. T., Rotteveel, M., et al. (2011). Convergent and divergent responses to emotional displays of ingroup and outgroup. Emotion, 11(2), 286-298. doi:10.1037/a0022582.

van der Weiden, A., Ruys, K. I., \& Aarts, H. (2013). A matter of matching: How goals and primes affect self-agency experiences. Journal of Experimental Psychology: General, 142(3), 954-966. https://doi.org/10.1037/a0030079

van Ulzen, N. R., Lamoth, C. J., Daffertshofer, A., Semin, G. R., \& Beek, P. J. (2008).

Characteristics of instructed and uninstructed interpersonal coordination while walking sideby-side. Neuroscience Letters, 432(2), 88-93. 
Vouloutsi, V., Grechuta, K., \& Verschure, P. F. (2019, July). Evaluation of the Facial Expressions of a Humanoid Robot. In Conference on Biomimetic and Biohybrid Systems (pp. 365-368). Springer, Cham.

Wass, S. V., Smith, C. G., Clackson, K., Gibb, C., Eitzenberger, J., \& Mirza, F. U. (2019). Parents mimic and influence their infant's autonomic state through dynamic affective state matching. Current Biology, 29(14), 2415-2422. https://doi.org/10.1016/ j.cub.2019.06.016

Waters, S. F., West, T. V., Karnilowicz, H. R., \& Mendes, W. B. (2017). Affect contagion between mothers and infants: Examining valence and touch. Journal of Experimental Psychology: General, 146(7), 1043.

Waters, S. F., West, T. V., \& Mendes, W. B. (2014). Stress contagion: Physiological covariation between mothers and infants. Psychological Science, 25(4), 934-942.

Wegner, D. M., \& Wheatley, T. (1999). Apparent mental causation: Sources of the experience of will. American Psychologist, 54(7), 480-492.

West, T. V., Koslov, K., Page-Gould, E., Major, B., \& Mendes, W. B. (2017). Contagious anxiety: Anxious European Americans can transmit their physiological reactivity to African Americans. Psychological Science, 28(12), 1796-1806.

Wheatley, T., Kang, O., Parkinson, C., \& Looser, C. E. (2012). From mind perception to mental connection: Synchrony as a mechanism for social understanding. Social and Personality Psychology Compass, 6(8), 589-606.

Woltering, S., Lishak, V., Elliott, B., Ferraro, L., \& Granic, I. (2015). Dyadic attunement and physiological synchrony during mother-child interactions: an exploratory study in children with and without externalizing behavior problems. Journal of Psychopathology and Behavioral Assessment, 37(4), 624-633. 
Wood, A., Rychlowska, M., Korb, S., \& Niedenthal, P. (2016). Fashioning the face: Sensorimotor simulation contributes to facial expression recognition. Trends in Cognitive Sciences, 20(3), 227-240.

Yun, K., Watanabe, K., \& Shimojo, S. (2012). Interpersonal body and neural synchronization as a marker of implicit social interaction. Scientific Reports, 2, 959.

Zhao, F., Wood, A., Mutlu, B., \& Niedenthal, P. (under review). Faces synchronize when communication through spoken language is prevented. 